\title{
Les torsions sur testicules cryptorchides
}

\author{
Mohamed Gharbi, MD; Najmeddine Amri, MD; Wahib Chambeh, MD; Salem Braiek, MD; Rafik El Kamel, MD
}

See related article on page 397.

\section{Résumé}

But : La cryptorchidie est une pathologie assez fréquente en urologie. Elle est associée à un risque élevé d'infertilité et de dégénérescence. Elle semble aussi être associée à un risque important de torsion. Cette entité est très peu abordée dans la littérature. Nous rapportons tous les cas de torsion sur testicule cryptorchide observés à notre service dans le but de mieux caractériser cette pathologie et de réduire ainsi le taux d'orchidectomies.

Méthodologie : Il s'agit d'une étude rétrospective portant sur tous les cas de torsion sur testicule cryptorchide opérés dans notre service d'urologie entre 1999 et 2007 . Les patients ont fait l'objet d'une description basée sur le résumé de leurs observations.

Résultats : Les patients étaient âgés de 7 mois à 39 ans. La torsion touchait le testicule droit dans $53 \%$ des cas. Le tableau clinique comportait une douleur au niveau de la région inguinale d'apparition soudaine avec une masse sous-cutanée inflammatoire et douloureuse à ce niveau et surtout un hémiscrotum homolatéral vide. Dans $60 \%$ des cas, le diagnostic était tardif et une orchidectomie a été réalisée. Dans les autre cas, un abaissement du testicule a été réalisé avec orchidopexie controlatéral dans le même temps opératoire.

Conclusion : Bien qu'il s'agisse d'une pathologie peu courante, la torsion sur testicule cryptorchide doit être étudiée davantage. Le diagnostic précoce permettra de sauver et d'abaisser le testicule et faciliter ainsi le dépistage d’une éventuelle dégénérescence.

Can Urol Assoc J 2010;4(6):393-6

\section{Abstract}

Purpose: Cryptorchidism is a rather frequent pathology in urology. It is associated with a high risk of infertility and degeneration. It also seems to be associated with a high risk of torsion. This entity is poorly studied in the literature. We studied the cases of torsion on cryptorchid testicles hospitalized at our service, to better characterize this pathology and reduce the rate of orchidectomy. Methods: We underwent a retrospective study of all the cases of torsion on a cryptorchid testicle hospitalized in our service of urology between 1999 and 2007.

Results: The age of patients was between 7 months and 39 years. Torsion touched the right testicle in $53 \%$ of the cases. Patients experienced a brutal pain of the inguinal area with an under cutaneous mass inflammatory and a painful and empty homolateral scrotum. In $60 \%$ of the cases, the diagnosis was late and an orchidectomy was carried out. In the other cases, a lowering of the testicle was done with the controlateral orchidopexy.

Conclusion: Even though torsions on a cryptorchid testicle are rare, we should not ignore them. The early diagnosis will make it possible to save and lower the testicle, and track a possible degeneration.

\section{Introduction}

La cryptorchidie est définie comme étant un défaut de migration du testicule par le canal inguinal vers le scrotum, plaçant le testicule en position ectopique dans ce canal ou en position intra-abdominale. L'incidence de la cryptorchidie chez les nouveau-nés à terme se situerait entre 2,7 et 5,9\%, alors qu'elle varie entre 9 et $30 \%$ chez les prématurés et est de $1,8 \%$ à l'âge $d^{\prime}$ un $a n^{1,2}$.

Les différentes publications portant sur cette pathologie sont axées sur le risque accru d'infertilité et de dégénérescence lié à cette anomalie ${ }^{3}$. Par ailleurs, la cryptorchidie est associée à un risque plus élevé de torsion par rapport à un testicule en place, risque qui serait 10 fois plus élevé selon les résultats signalés par Williamson ${ }^{4}$. II s'agit d'une véritable urgence urologique et le diagnostic est souvent retardé vu le siège inhabituel du testicule et est donc associé à un risque plus élevé d'orchidectomie.

Nous rapportons, dans cette série, tous les cas de torsion sur testicule cryptorchide admis dans notre service d'urologie dans le but de mieux caractériser cette entité clinique pour ainsi faciliter le diagnostic et d'essayer par le fait même de réduire le taux d'orchidectomies.

\section{Patients et méthodologie}

Il s'agit d'une étude rétrospective portant sur tous les cas de torsion sur testicule cryptorchide opérés dans notre service d'urologie de Kairouan (Tunisie) sur 8 ans entre 1999 et 2007.

Les données ont été tirées des dossiers des patients, des fichiers du service des urgences et des comptes rendus opératoires. 
Cette étude ne pose aucun problème d'éthique et a été autorisée par notre comité d'éthique.

\section{Résultats}

Entre 1999 et 2007, quinze patients présentant un tableau clinique de torsion sur testicule cryptorchide ont été opérés à notre service. L'âge des patients variait entre 7 mois et 39 ans. Douze patients avaient moins de 15 ans et les trois autres, plus de 20 ans. Chez deux enfants, le diagnostic n'a pas été posé immédiatement aux urgences et ils ont été hospitalisés à tort au service de chirurgie générale en raison d'une collection sous-cutanée soupçonnée dans la région inguinale. Trois patients avaient une cryptorchidie bilatérale. La torsion touchait le testicule droit dans $53 \%$ des cas.

Chez la majorité des patients de moins de 15 ans, la cryptorchidie n'avait pas été décelée par les parents (10 cas sur 12 , soit $83,3 \%$ des cas). Dans les deux autres cas, les parents avaient décelé la cryptorchidie mais n'avaient pas consulté le médecin, par manque de connaissances.

Le tableau clinique comportait dans la majorité des cas une douleur au niveau du canal inguinal avec à l'examen clinique une masse sous-cutanée douloureuse accompagnée de signes inflammatoires locorégionaux (chaleur et rougeur). Des symptômes digestifs (nausées et vomissements) ont été observés chez 7 patients, soit dans 46,6\% des cas. Ces symptômes digestifs étaient très significatifs et dominaient le tableau clinique chez un patient présentant une torsion sur un testicule intra-abdominal. Quatre patients ont été admis dans les 6 heures après l'apparition des symptômes.

Dans un cas, le patient était âgé de 39 ans et il a consulté en raison d'un tableau clinique comprenant des douleurs péri-ombilicales et au niveau de la fosse iliaque droite avec notion de cryptorchidie droite. Le patient a été opéré $d^{\prime}$ urgence en raison d'un diagnostic de torsion sur testicule cryptorchide droit. L'exploration chirurgicale a permis d'objectiver un testicule tumoral intra-péritonéal tordu. Une orchidectomie a été réalisée.

Chez un seul enfant, une échographie Doppler a permis de conclure à un testicule inguinal atrophique avec absence de perfusion du parenchyme testiculaire.

L'exploration a été effectuée dans tous les cas par incision inguinale. Le testicule était d'aspect nécrosé évident dans 9 cas sur 15 , soit chez $60 \%$ des patients, et une orchidectomie a été réalisée. Dans les autres cas, le testicule a été récupéré après détorsion et un abaissement dans le même temps opératoire a été réalisé. Une orchidopexie du testicule controlatéral a été réalisée dans le même temps opératoire chez les patients ayant une cryptorchidie unilatérale. Aucune complication n'a été signalée pendant la période postopératoire.

Dans un seul cas, l'examen anatomopathologique a conclu à un testicule tumoral, soit chez le patient avec un testicule intra-abdominal tordu (séminome pur).
Le suivi après deux ans chez les patients ayant eu une conservation de leur testicule (6 cas) a révélé un cas de testicule de taille et morphologie normales identiques au testicule controlatéral, trois cas de testicule de morphologie normale mais de taille inférieure à l'autre testicule et deux cas d'atrophie testiculaire.

\section{Discussion}

L'étude de la cryptorchidie et des ectopies testiculaires a débuté vers le $18^{\mathrm{e}}$ siècle avec les travaux du baron Albrecht Von Haller et de John Hunter. II s'agissait de descriptions purement anatomiques sur des cadavres. Les premières théories sur le défaut de migration des testicules de la cavité abdominale vers le scrotum lors de la vie fœtale ont été rapportées par Curling un siècle plus tard ${ }^{5}$.

La cryptorchidie est une pathologie congénitale assez fréquente en urologie pédiatrique. Ce risque est multiplié par 10 chez les prématurés, les nouveau-nés ayant un faible poids à la naissance et ceux atteints d'une pathologie neuromusculaire ${ }^{6}$.

Ce défaut de migration interfère avec le processus de maturation des gonocytes néonatals en spermatogonies de type A. L'absence de cette différenciation est à l'origine des complications liées à cette pathologie, dont les plus documentées dans la littérature sont l'infertilité et la transformation maligne ${ }^{3}$. Le taux de fertilité est de $14 \%$ chez les sujets traités après l'âge de 13 ans pour une cryptorchidie bilatérale contre $87,5 \%$ chez ceux opérés avant l'âge de 2 ans $^{7}$.

La cryptorchidie représente le principal facteur de risque du cancer testiculaire. Ce risque varie de 2,5 à $18 \%$. Il est maximal en cas de cryptorchidie bilatérale et n'est pas réduit quel que soit l'âge lors de l'abaissement testiculaire ${ }^{8}$.

L'absence du testicule dans le scrotum prédispose également à d'autres risques, à savoir un risque élevé de torsion et une exposition aux traumatismes, sans oublier l'impact psychologique de cette pathologie.

On a récemment décrit une autre forme de cryptorchidie survenant à un âge plus avancé, soit une cryptorchidie secondaire ou acquise, dont l'incidence est de 1 à $2 \%$. Cette pathologie est due à un défaut d'élongation du cordon spermatique pendant la croissance secondaire à un remaniement fibreux dans le cordon spermatique. Cliniquement, on aura une migration secondaire du testicule du scrotum vers le canal inguinal. L'étiologie est encore mal élucidée ${ }^{9}$.

La torsion du cordon spermatique a été décrite pour la $1^{\text {re }}$ fois par Delasiauve en 1840 chez un garçon de 15 ans présentant une torsion sur un testicule en position inguinale. Le patient a subi une orchidectomie ${ }^{10}$. En 1857, Curling décrit un cas similaire mais sur un testicule viable; le patient a subi une détorsion suivie d'un abaissement et d'une orchidopexie ${ }^{11}$. 
Une revue des articles publiés sur un siècle montre que cette pathologie n'a pas été bien étudiée et que peu d'auteurs se sont intéressés à cette entité clinique. Seuls quelques cas ont été publiés dans les annales des urgences. Un article récent fait état d'une série de 11 cas recueillis au département d'urologie du Chaim Sheba Medical Center à Tel Aviv ${ }^{12}$. Dans notre série, nous rapportons une série de 15 cas sur 8 ans.

Dans les différents articles publiés, la cryptorchidie était associée à un risque plus élevé de torsion par rapport au testicule en place. En effet, dans la série de Johnson, sur les $7 \%$ de cas de cryptorchidies décrits à l'âge néonatal, $23 \%$ présentaient une torsion ${ }^{13}$. Williamson estime que le risque de torsion est 10 fois plus élevé sur un testicule cryptorchide 4 .

Le mécanisme de torsion dans ce contexte reste mal connu. Une des théories parle d'une disproportion entre la taille du cordon et la taille du testicule cryptorchide, qui est souvent atrophique, créant ainsi une plus forte tension sur le testicule et entraînant donc un risque élevé de torsion. Cette théorie est également valable lorsqu'on a un testicule tumoral qui peut se tordre sous l'effet de son propre poids ${ }^{14}$. Une $2^{\mathrm{e}}$ théorie implique le rôle du muscle crémaster qui, par des contractions pathologiques, peut induire des torsions chez les sujets souffrant $d^{\prime}$ une pathologie neuromusculaire ${ }^{15}$. Une $3^{\text {e }}$ théorie implique un défaut $d^{\prime}$ apposition de l'épididyme par rapport au testicule, créant une configuration particulière prédisposant à la torsion ${ }^{3}$. La torsion est deux fois plus importante quand il s'agit du testicule gauche car le cordon dans ce cas est plus long ${ }^{16}$.

Une torsion sur testicule cryptorchide doit être soupçonnée chez tout enfant consultant pour une douleur inguinale ou abdominale avec une masse inguinale douloureuse et un hémiscrotum homolatéral vide ${ }^{14}$.

Dans notre série, un seul patient avait subi une échographie Doppler. Cet examen est largement recommandé dans la littérature. Dans les cas typiques, il révèle un testicule tuméfié avec absence de vascularisation ${ }^{3,12}$. Cet examen complémentaire ne doit en aucun cas retarder l'exploration chirurgicale qui seule pourra sauver le testicule.

La torsion d'un testicule intra-abdominal est courante chez I'adulte de 20 à 40 ans. Le tableau clinique comprend des douleurs abdominales avec symptômes digestifs (nausées, vomissements). Les diagnostics différentiels les plus fréquents sont l'appendicite aiguë et les diverticulites, mais l'absence de testicule homolatéral est un bon indicateur diagnostique. Le risque de dégénérescence dans cette localisation est 4 fois plus important qu'avec un testicule inguinal ${ }^{17,18}$.

$\mathrm{Si}$ le testicule est viable après détorsion, les auteurs préconisent deux options différentes, c'est-à-dire un abaissement soit dans le même temps opératoire, soit dans un second temps.
La majorité des auteurs recommandent la fixation systématique du testicule controlatéral ${ }^{3,12,14}$.

Le taux de préservation du testicule tordu en position scrotale varie entre 20 et $92 \%$ selon les séries ${ }^{12}$. La littérature ne fournit aucun taux dans les cas de cryptorchidie, mais il semble que les chances de récupération soient moindres vu que le diagnostic est souvent fait tardivement.

\section{Conclusion}

La torsion testiculaire en présence de cryptorchidie est un incident relativement rare mais qui devrait faire partie des diagnostics différentiels d'une masse inguinale, surtout chez des patients ayant une pathologie neuromusculaire spastique. La familiarisation avec cette pathologie pourra aider à poser le diagnostic plus tôt et permettre ainsi de sauver le testicule tordu. La chirurgie en urgence est le seul traitement à proposer. L'orchidopexie du testicule controlatéral devrait être systématique dans le même temps opératoire ou ultérieurement. Le diagnostic précoce de la cryptorchidie par le dépistage systématique à la naissance et en milieu scolaire reste le seul moyen de lutter contre cette pathologie.

Unité chirurgicale « Les Aghlabides », service d'urologie, Kairouan, Tunisie

Competing interests: None declared.

This paper has been peer-reviewed.

\section{Bibliographie}

1. Pillai SB, Besner GE. Pediatric testicular problems. Pediatr Clin North Am 1998;45:813.

2. Scorer CG. The descent of the testis. Arch Dis Child 1964;39:605-9.

3. Hutson JM, Clarke MC. Current management of the undescended testicle. Sem Ped Surg 2007;16:64-70.

4. Williamson RCN. Torsion of the testis and allied conditions. Br I Surg 1976;63:465.

5. Tackett LD, Patel SR, Caldamone AA. A history of cryptorchidism: Lessons from the eighteenth century. J Ped Urol 2007;3:426-32.

6. Cilento BG, Najiar SS, Atala A. Cryptorchidism and testicular torsion. Pediatr Clin North Am 1993;40:1133.

7. Mackinnon AE. The undescended testis. Indian J Pediatr 2005;72:429-32.

8. Mottet N. Facteurs de risque du cancer du testicule et mécanisme de la carcinogénèse. Prog Urol 2003;13:1244-5.

9. Hutson JM, Goh DW. Can undescended testes be acquired? Lancet 1993;341:504.

10. Schultz KE, Walker J. Testicular torsion in undescended testes. Ann Emerg Med 1984;13:567.

11. Noske HD, Kraus SW, Altinkilic BM, et al. Historical milestones regarding torsion of the scrotal organs. J Urol 1998;159:13.

12. Zilberman D, Inbar Y, Heyman Z, et al. Torsion of the cryptorchid testis. Can it be salvaged? I Urol 2006; $175: 2287-9$

13. Johnson JH. The undescended testis. Arch Dis Child 1965;40:113.

14. Schneck FX, Bellinger MF. Abnormalities of the testis and scrotum and their surgical management. In: Campbell's Urology. 8th ed. P.C. Walsh, A.B. Retik, E.D. Vaughan Jr., A.J. Wein (editors). Philadelphia, PA: W.B. Saunders C0., 2002:2353-94.

15. Schultz KE. Walker J. Testicular Torsion in Undescended Testes. Ann Emerg Med 1984;13:7. 
Gharbi et al.

16. Ein SH. Torsion of an Undescended Intrababdominal Benign Testicular Teratoma. J Ped Surg 1987;22:799801.

17. Lewis RL, Roller MD, Parra BL, et al. Torsion of an Intra-Abdominal Testis. Current Surg 2000;57:497-9.

Correspondence: Dr Mohamed Gharbi, BP 105, Kairouan 3100, Tunisie ; mohamed_gharbi_tn@

18. Riegler HC. Torsion of intra-abdominal testis. Surg Clin North Am 1985;65:1303-29.

\section{For the latest on CUAJ, subscribe to the electronic table of contents.}

Send an email to journal@ cua.org with the word SUBSCRIBE in the subject line. 